\title{
Agile Health Care Analytics: Enabling Real-Time Disease Surveillance With a Computational Health Platform
}

Wade L Schulz ${ }^{1,2}$, MD, PhD; Thomas J S Durant ${ }^{1,2}$, MD; Charles J Torre Jr ${ }^{1,3}$, MS; Allen L Hsiao ${ }^{3,4}$, MD; Harlan M Krumholz $^{2,5,6}$, SM, MD

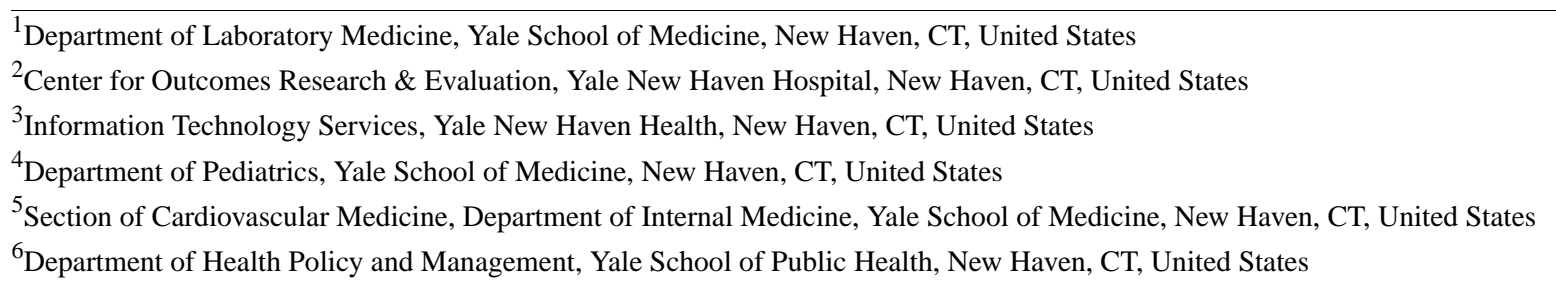

Corresponding Author:

Wade L Schulz, MD, PhD

Department of Laboratory Medicine

Yale School of Medicine

55 Park St, PS215

New Haven, CT, 06511

United States

Phone: 1 (203) 6882286

Email: wade.schulz@yale.edu

\section{Abstract}

The ongoing coronavirus disease outbreak demonstrates the need for novel applications of real-time data to produce timely information about incident cases. Using health information technology (HIT) and real-world data, we sought to produce an interface that could, in near real time, identify patients presenting with suspected respiratory tract infection and enable monitoring of test results related to specific pathogens, including severe acute respiratory syndrome coronavirus 2 . This tool was built upon our computational health platform, which provides access to near real-time data from disparate HIT sources across our health system. This combination of technology allowed us to rapidly prototype, iterate, and deploy a platform to support a cohesive organizational response to a rapidly evolving outbreak. Platforms that allow for agile analytics are needed to keep pace with evolving needs within the health care system.

(J Med Internet Res 2020;22(5):e18707) doi: $\underline{10.2196 / 18707}$

\section{KEYWORDS}

real-time analytics; real-world data; disease surveillance; computational health; surveillance; public health; COVID-19; outbreak; health information technology; HIT; interface; monitoring; pandemic

The ongoing coronavirus disease (COVID-19) outbreak demonstrates the need for novel applications of real-time data to produce timely information about incident cases [1]. Using health information technology (HIT) and real-world data (RWD), we sought to produce an interface that could in near real time, identify patients presenting with suspected respiratory tract infection and enable monitoring of test results related to specific pathogens, including severe acute respiratory syndrome coronavirus 2 (SARS-CoV-2).

This tool was built upon our computational health platform (CHP), which provides a data integration and analysis platform across our health system [2]. The platform enriches clinical data with geolocation information and real-time data from our enterprise integration engine. Data within our clinical data warehouses are transformed into the Observational Medical Outcomes Partnership (OMOP) common data model [3] and merged with data from the integration engine to support real-time analytics and promote the capacity for rapid collaboration. We developed an interactive dashboard to summarize these data and present them in a format suitable for analysis (Figure 1). 
Figure 1. The architecture of data flows within the computational health platform with key integration points that allow for real-time data access and agile analytics. HL7: Health Level 7.

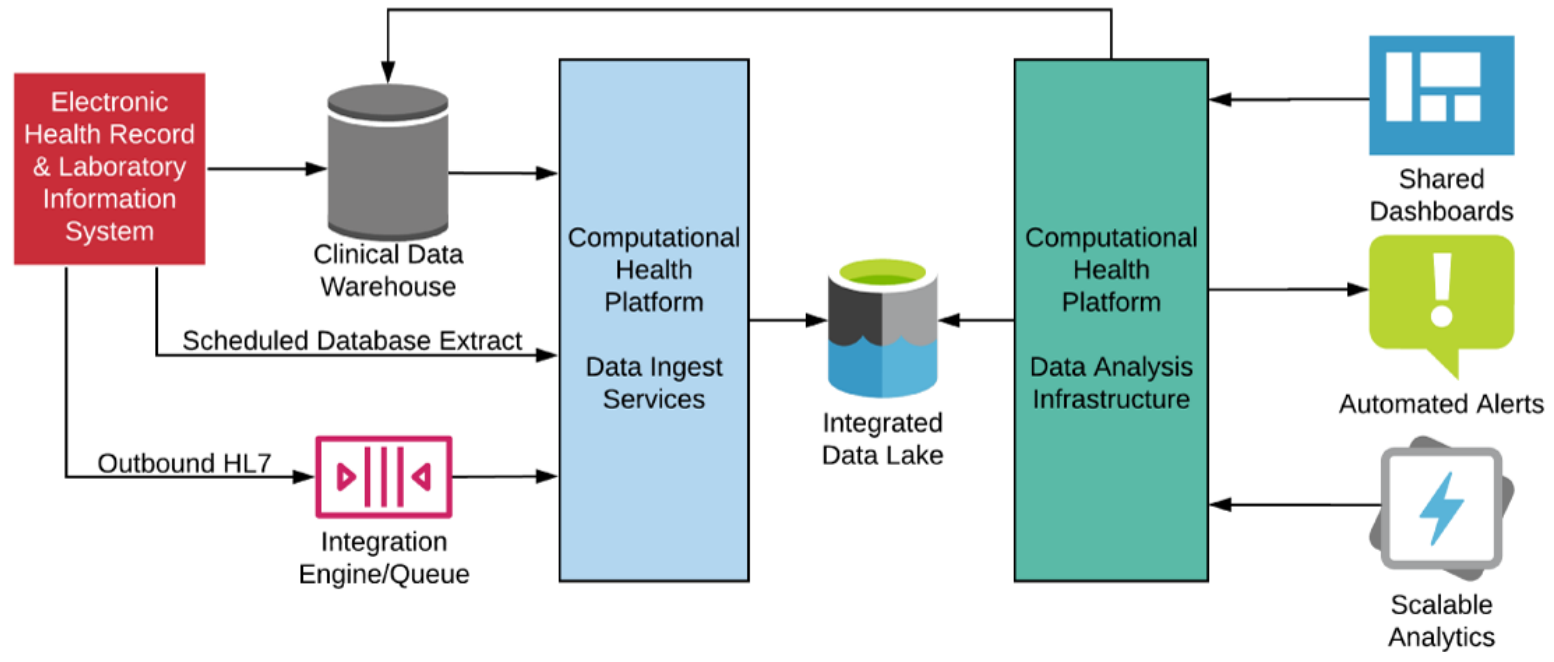

Factors impeding data access included limitations in electronic health record (EHR) interfaces and manual reporting workflows. After moving to an integrated EHR and laboratory information system, our Health Level 7 order and results feed became limited to results, which prevented access to other essential data such as order information, requiring us to develop a method to extract data from the underlying clinical database. The second bottleneck was the manual workflow in reporting COVID-19 results from the state reference laboratory, which initially relied on faxed paper results.

This real-time platform provides several benefits for managing a cohesive organizational response to a rapidly evolving outbreak. Trends can be tracked, issues with individual patients can be identified, hot spots can be determined, and new patients can be automatically reported for contact tracing. Delays in testing and resulting can also be identified across an integrated health care delivery system to address potential barriers to care delivery. Since these data are integrated with our OMOP data repository, downstream apps and timely observational research are also supported.

From March 1, 2020, through May 14, 2020, the tools described here were used to follow over 40,000 patients tested for COVID-19 across our health system, with information regarding admissions, intensive care unit capacity, and ventilator use for over 9600 patients with positive SARS-CoV-2 results. Dashboards were created to track laboratory testing volumes, turnaround times, and outstanding tests. The platform was also used to create a clinical COVID-19 data registry to support operational analytics, quality improvement initiatives, and biomedical research across the organization, and to create clinical predictive models [4].
With the rapid increase in COVID-19 and associated morbidity and mortality, there is a noted urgency to leverage available resources to identify risk factors and possible treatments, and track outcomes. For this, high-quality data are needed to generate evidence [5]. Although randomized controlled trials are being implemented internationally, they have several limitations, including typically long time periods from initiation to results. As such, other methods are needed to provide short-term information that can be used to guide the clinical and organizational response to a rapidly evolving pandemic. RWD has the potential to offer important insights into the management of COVID-19 and for the development of clinical predictive models that can be used in frontline care. Many investigators have begun to take advantage of RWD for COVID-19 research, but, although the information generated from RWD sources can be valuable, ensuring high-quality analysis requires new methodologic approaches, significant data validation, and careful interpretation of results.

Platforms that allow for agile analytics are needed to keep pace with evolving needs within the health care system. By leveraging our existing CHP, the initial tool was created and sourced with near real-time data in approximately 1 day of development effort from one developer and one clinical informaticist. These systems require an integrated team of HIT experts, clinical informaticists, health care providers, and administrative support to ensure that high-quality data can be efficiently disseminated across the enterprise. Such systems can be used to generate the RWD needed to drive evidence generation, especially with a rapidly evolving pandemic, but care is needed to ensure high-quality data are produced and that results are correctly interpreted given their observational nature.

\section{Acknowledgments}

We would like to acknowledge Nathan Price, William Byron, and Patrick Young for their assistance in developing the underlying platform and data feeds along with the ongoing updates to the analytic dashboard. We would like to acknowledge Phil Corso and Brian Keane for their assistance with ongoing analytic dashboard design and support. 


\section{Authors' Contributions}

WS and TD conceived the study design, performed the analysis, and drafted and revised the manuscript. CT conceived the study design and revised the manuscript. AH and HK assisted with the study design and provided critical revision of the manuscript.

\section{Conflicts of Interest}

HK works under contract with the Centers for Medicare \& Medicaid Services to support quality measurement programs; was a recipient of a research grant, through Yale University, from Medtronic and the US Food and Drug Administration to develop methods for postmarket surveillance of medical devices; was a recipient of a research grant with Medtronic and is the recipient of a research grant from Johnson \& Johnson, through Yale University, to support clinical trial data sharing; was a recipient of a research agreement, through Yale University, from the Shenzhen Center for Health Information for work to advance intelligent disease prevention and health promotion; collaborates with the National Center for Cardiovascular Diseases in Beijing; receives payment from the Arnold \& Porter Law Firm for work related to the Sanofi clopidogrel litigation, the Ben C Martin Law Firm for work related to the Cook Celect IVC filter litigation, and the Siegfried and Jensen Law Firm for work related to Vioxx litigation; chairs a Cardiac Scientific Advisory Board for UnitedHealth; was a participant/participant representative of the IBM Watson Health Life Sciences Board; is a member of the Advisory Board for Element Science, the Advisory Board for Facebook, and the Physician Advisory Board for Aetna; and is the cofounder of the personal health information platform HugoHealth and cofounder of Refactor Health, an AI-augmented data management platform for health care. WS was an investigator for a research agreement, through Yale University, from the Shenzhen Center for Health Information for work to advance intelligent disease prevention and health promotion; collaborates with the National Center for Cardiovascular Diseases in Beijing; is a technical consultant to HugoHealth, a personal health information platform, and cofounder of Refactor Health, an AI-augmented data management platform for health care; and is a consultant for Interpace Diagnostics Group, a molecular diagnostics company. The other coauthors report no potential conflicts of interest.

\section{References}

1. Dong E, Du H, Gardner L. An interactive web-based dashboard to track COVID-19 in real time. Lancet Infect Dis 2020 May;20(5):533-534. [doi: 10.1016/s1473-3099(20)30120-1]

2. McPadden J, Durant TJ, Bunch DR, Coppi A, Price N, Rodgerson K, et al. Health care and precision medicine research: analysis of a scalable data science platform. J Med Internet Res 2019 Apr 09;21(4):e13043. [doi: 10.2196/13043] [Medline: 30964441]

3. Observational Health Data Sciences and Informatics. OMOP common data model URL: https://www.ohdsi.org/ data-standardization/the-common-data-model/ [accessed 2019-03-02]

4. Haimovich A, Ravindra NG, Stoytchev S, Young HP, Wilson FP, van Dijk D, et al. Development and validation of the COVID-19 severity index (CSI): a prognostic tool for early respiratory decompensation. medRxiv 2020 May 14:a. [doi: 10.1101/2020.05.07.20094573]

5. Hope AA, Munro CL. Discussing evidence in the fog of the pandemic. Am J Crit Care 2020 May 05:e1-e3. [doi: 10.4037/ajcc2020239] [Medline: 32369547]

\section{Abbreviations}

CHP: computational health platform

COVID-19: coronavirus disease

EHR: electronic health record

HIT: health information technology

OMOP: Observational Medical Outcomes Partnership

RWD: real-world data

SARS-CoV-2: severe acute respiratory syndrome coronavirus 2

Edited by G Eysenbach; submitted 12.03.20; peer-reviewed by T Ueno, A Benis; comments to author 20.04.20; revised version received 16.05.20; accepted 20.05.20; published 28.05.20

Please cite as:

Schulz WL, Durant TJS, Torre Jr CJ, Hsiao AL, Krumholz HM

Agile Health Care Analytics: Enabling Real-Time Disease Surveillance With a Computational Health Platform

J Med Internet Res 2020;22(5):e18707

URL: http://www.jmir.org/2020/5/e18707/

doi: $10.2196 / 18707$

PMID: 32442130 
(C) Wade L Schulz, Thomas J S Durant, Charles J Torre Jr, Allen L Hsiao, Harlan M Krumholz. Originally published in the Journal of Medical Internet Research (http://www.jmir.org), 28.05.2020. This is an open-access article distributed under the terms of the Creative Commons Attribution License (https://creativecommons.org/licenses/by/4.0/), which permits unrestricted use, distribution, and reproduction in any medium, provided the original work, first published in the Journal of Medical Internet Research, is properly cited. The complete bibliographic information, a link to the original publication on http://www.jmir.org/, as well as this copyright and license information must be included. 